\title{
Sozialpädagogische oder psychosoziale Prozessbegleitung - eine Optimierung des Opferschutzes im Strafverfahren
}

Wahrheitsermittlung und Ahndung von Straftaten als Teil des staatlichen Auftrags zur Strafverfolgung, dem Verfassungsrang zukommt, sind Grundprinzipien und Ideale, die nicht immer den Interessen der beteiligten Menschen, insbesondere der Opfer, Rechnung tragen. Zwar ist nach jahrzehntelanger Diskussion nunmehr unstreitig, dass nicht nur dem beschuldigten Tatverdächtigen im Strafverfahren, sondern auch dem Opfer, also der oder dem Verletzten als Zeugin oder Zeuge die Beachtung der Grundrechte, insbesondere des Rechts auf Menschenwürde zustehen.

Auch aus diesen Gründen wurden in den letzten Jahren die Rechte der Verletzten im Strafverfahren durch mehrere Reformen der Strafprozessordnung (StPO) gestärkt. Die dabei konzipierte Doppelunterstützung durch Verletztenbeistand $(\mathbb{S}$ 68b, $406 \mathrm{f}$ StPO) und anwaltliche Nebenklagevertretung ( $\mathbb{S}$ 406g StPO) war dabei grundsätzlich gut gemeint. Mangels erforderlicher Professionalität insbesondere bei dem Verletztenbeistand ist jedoch besonders bei kindlichen oder jugendlichen Opferzeugen oder bei traumatisierten Verletzten der Minimierung der Belastungen im Strafverfahren nicht in ausreichendem Maße genügt worden. Die Unterstützung war insbesondere im Hinblick auf die pädagogischen Erfordernisse der betroffenen kindlichen oder jugendlichen Opferzeuginnen und Opferzeugen also nicht (immer) gut. Eine professionelle Schulung im Hinblick auf traumatisierte Zeugen ist zudem extrem selten. Um die Aussageleistung im Ermittlungs- und Strafverfahren gerade dieser Verletzten bei Wahrung der notwendigen professionellen Distanz zu stabilisieren und insbesondere ihre seelische Gesundheit zu wahren, bedarf es deshalb einer grundlegenden Professionalisierung der Begleitung von insbesondere kindlichen und jugendlichen und traumatisierten Verletzten im gesamten Strafverfahren.

Professionelle Begleitung umfasst dabei nicht nur die Anwesenheit bei Vernehmungsterminen. Sie bedeutet auch, den Opfern nicht verstandene Verfahrensnotwendigkeiten, wie zum Beispiel wiederholte Vernehmungen oder durchzuführende, gegebenenfalls die Intimsphäre berührende Untersuchungen zu erläutern oder insbesondere Gefährdungen für das seelische Wohl und die weitere Ent- wicklung des Opfers frühzeitig zu erkennen und insoweit vorbeugend tätig zu werden. Wenn es sich bei den Opfern um Kinder oder Jugendliche handelt, bedarf es zudem besonderer Kenntnisse aus dem Bereich der Pädagogik und der Entwicklungspsychologie, bei traumatisierten Opfern der Kenntnis der Phänomenologie und daraus folgenden Reaktionsweisen des Traumas und zusätzlicher Risiken durch verfahrensbedingte Notwendigkeiten wie Untersuchungen und Vernehmungen. Dass der Schutz der Opfer vor derartigen - zusätzlichen Belastungen nicht dem Zufall der Anwesenheit einer entsprechend vorgebildeten ehrenamtlichen Prozessbegleitung überlassen werden sollte, liegt auf der Hand. Eine entsprechende Professionalisierung durch im Idealfall zertifizierte Fortbildung ist angezeigt und wird in Modellprojekten ${ }^{1}$, unterstützt durch das Bundesministerium der Justiz, angeboten. Dieses hatte nunmehr zu einem Gedankenaustausch über die Notwendigkeit einer professionalisierten sozialpädagogischen oder psychosozialen Prozessbegleitung und deren Verankerung im Gesetz zahlreiche Berufsgruppen und Nichtregierungsorganisationen, aber auch betroffene $\mathrm{Mi}$ nisterien und Mitglieder anderer öffentlicher Einrichtungen eingeladen.

Dem Grundgedanken der Erforderlichkeit der im vorgenannten Sinne professionalisierten Prozessbegleitung haben sich dabei alle Eingeladenen im Ergebnis angeschlossen, nachdem Einigkeit darüber bestand, dass die in Rede stehenden Tätigkeiten von den vorhandenen Verfahrensbeteiligten nicht geleistet werden können. Richterin oder Richter und Staatsanwältin oder Staatsanwalt scheiden bereits aufgrund der ihnen gebotenen Objektivität für eine derartige, eher parteigebundene Aufgabe aus. In Fällen der zuzulassenden oder zugelassenen Nebenklage gem. $\mathbb{S}$ 406g, 397a, 395 StPO stehen im Ermittlungsverfahren die für die kindlichen und jugendlichen Opferzeuginnen oder Opferzeugen be-

1 Achtmonatige interdisziplinäre berufsbegleitende Weiterbildung für (sozial-)pädagogische Fachkräfte zur sozialpädagogischen Prozessbegleiterin/zum sozialpädagogischen Prozessbegleiter für verletzte Zeuginnen und Zeugen im Strafverfahren des Instituts für Opferschutz e.V. „Recht Würde Helfen“ unter der Schirmherrschaft von Bundesjustizministerin Zypries; Informationen unter www.rwh-institut.de.

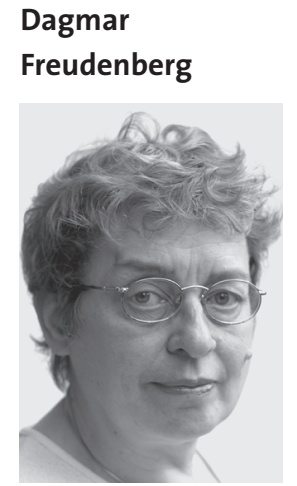

Vorsitzende der Kommission Gewalt gegen Frauen und Kinder des djb; Vorsitzende der Regionalgruppe Göttingen im djb; Staatsanwältin im Sonderdezernat häusliche Gewalt und Sonderdezernat Sexualstraftaten, Göttingen 
auftragten Nebenklagevertreterinnen oder Nebenklagevertreter für Unterstützung, Begleitung, Erklärung und Trost zur Verfügung. Hauptaufgabe dieses juristischen Beistandes ist jedoch, die Wahrung der Beteiligungs- und Informationsrechte der kindlichen und jugendlichen Verletzten zu gewährleisten. Nebenklageanwältin und Nebenklageanwalt müssen an der Hauptverhandlung zur Wahrung der Rechte ihrer Mandantinnen und Mandanten von Beginn an teilnehmen, insbesondere auch während der Einlassung der oder des Angeklagten zur Person oder zur Sache. Zwar haben auch die Verletzten das Recht, selbst an der gesamten Beweisaufnahme, also auch während der Aussage der oder des Angeklagten, anwesend zu sein. Aus Gründen ihrer Glaubwürdigkeit ist dies jedoch regelmäßig kontraproduktiv. Warten sie indes ihren Aufruf als Zeugin oder Zeuge außerhalb des Gerichtssaals ab, stehen ihnen Nebenklagevertreterin oder Nebenklagevertreter für ihre Begleitung nicht zur Verfügung. Vor allem aber sind die Nebenklageanwältin und der Nebenklageanwalt für sozialpädagogische oder psychosoziale Begleitung bei potentiell traumatisierten Zeuginnen oder Zeugen zumeist nicht ausgebildet. Die Aufgabe muss deshalb nach dem Ergebnis der Diskussion Prozessbegleiterinnen oder Prozessbegleitern übertragen werden. ${ }^{2}$ Dabei wurde aber zugleich festgestellt, dass es der Erarbeitung von Standards für die Durchführung der Prozessbegleitung ebenso bedarf wie für die Durchführung der Aus- bzw. Fortbildung dieser Prozessbegleiterinnen und Prozessbegleiter. Zudem wird zu prüfen sein, ob das Angebot sozialpädagogischer oder psychosozialer Prozessbegleitung nur bestimmten Gruppen von Opfern angeboten werden sollte, wie zum Beispiel kindlichen und jugendlichen Opfern von Straftaten oder generell Opfern von Gewalttaten oder besonders schweren Straftaten. Wie schlussendlich eine gesetzliche Verankerung in der Strafprozessordnung erfolgen kann, wird im Hinblick auf die Frage der Kostenübernahme durch den Staat oder durch den Verurteilten noch weiter zu diskutieren sein.

Siehe auch Stellungnahme des Deutschen Juristinnenbundes (djb)
St 07-21 Psychosoziale Prozessbegleitung unter www.djb.de/ stellungnahmen-und-pressemitteilungen/.

\title{
Nebenklage: im Sinne der Opfer und der Wahrheitsermittlung
}

\author{
Bewertung zweier aktueller Gesetzentwürfe
}

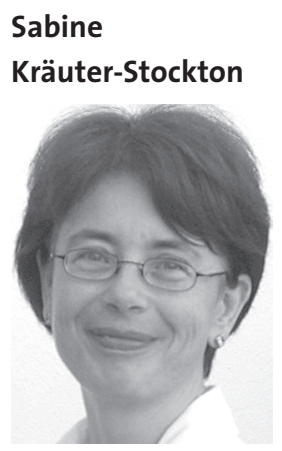

Mitglied der Kommission Gewalt gegen Frauen und Kinder des djb; Vorsitzende des Landesverbands Saarland im djb; Staatsanwältin im Sonderdezernat Häusliche Gewalt und Sexuelle Gewalt, zuvor Koordinierungsstelle gegen Häusliche Gewalt beim Saarländischen Justizministerium, Saarbrücken
Seit Jahrzehnten versteht es der djb als seine Aufgabe, das Augenmerk auf die - heute nach wie vor bestehende - Notwendigkeit zu lenken, die Stellung von Zeuginnen und Zeugen, insbesondere von Verletzten im Strafverfahren zu verbessern ${ }^{1}$. Das Thema ist jetzt wieder einmal hoch aktuell: Im Bundesrat werden zwei Gesetzentwürfe diskutiert, die unter dem Gesichtspunkt des Zeugenschutzes eingebracht wurden. In dem einen wird vorgeschlagen, die Nebenklagebefugnis mit der Möglichkeit einer kostenlosen Opferanwältin oder eines kostenlosen Opferanwalts auf Opfer von Zwangsheirat und von schwerem „Stalking“ zu erstrecken ${ }^{2}$, der andere will die Beiordnung einer Opferanwältin oder eines Opferanwalts für die bereits nach jetziger Rechtslage nebenklageberechtigten Opfer von schweren Körperverletzungen und von erpresserischem Menschenraub sowie von Geiselnahme ermöglichen ${ }^{3}$.

\section{Warum Zeugenschutz?}

Das Erfordernis des Zeugenschutzes folgt grundsätzlich aus der staatlichen Schutzpflicht gegenüber Beeinträchtigungen von Leben und körperlicher Unversehrtheit, aus der Pflicht zur Achtung der Per- sönlichkeit und der gerichtlichen und staatsanwaltschaftlichen Fürsorgepflicht für Zeuginnen und Zeugen ${ }^{4}$. Zeugenschutz war zwar schon in den Anfängen der deutschen Prozessrechtskodifizierung ein Thema ${ }^{5}$, wurde aber erst in den 1980er Jahren Gegenstand eingehender kriminalpolitischer Erörterungen, die einen Perspektivwechsel hinsichtlich der Stellung von Zeuginnen und Zeugen und insbesondere von Opferzeuginnen und Opferzeugen im

1 U.a. Stellungnahme zum Entwurf eines Ersten Gesetzes zur Verbesserung der Stellung von Verletzten im Strafverfahren vom 15.5.1986 (Archiv des djb); Fachtagung des djb vom 30.9.-2.10.1994 in der Berliner Humboldt-Universität zur Verbesserung der Situation von Opfern sexueller Gewalt; Fachtagung des djb in Bonn vom 27.2.1998 zur Reform der Nebenklage; Nelles, U./Oberlies, D.: Reform der Nebenklage und anderer Verletztenrechte, Nomos 1998.

2 BR Drs 872/07.

3 BR Drs 656/07.

4 Löwe/Riess: Die StPO und das GVG, Großkommentar, Einleitung Abschn. I Fn 127.

5 Zeugenschutz wurde schon im 19. Jahrhundert in den Beratungen zur Einführung der StPO diskutiert; siehe hierzu: Christina Ullrich, Schutz des verletzten Zeugen durch Entfernung des Angeklagten gem. § 247 StPO im Bereich der Sexualdelinquenz, LIT-Verlag Berlin-Hamburg-Münster 1998, S. 19-21. 